Article

\title{
Half-Metallic Properties of Single-Walled Polymeric Manganese Phthalocyanine Nanotubes
}

Hongbin Jiang ${ }^{1,2}$, Meilin Bai ${ }^{2}$, Peng Wei ${ }^{2}$, Lili Sun ${ }^{2}$, Ziyong Shen ${ }^{2}$ and Shimin Hou ${ }^{2, *}$

1 School of Physics \& Electronic Engineering, Sichuang Mianyang Normal University, Mianyang 621000, China; E-Mail: 271479861@qq.com

2 Key Laboratory for the Physics and Chemistry of Nanodevices, Department of Electronics, Peking University, Beijing 100871, China; E-Mails: meilin.bai@gmail.com (M.B.); weipeng.cumt@163.com (P.W.); sunlili.cb@gmail.com (L.S.); zyshen@pku.edu.cn (Z.S.)

* Author to whom correspondence should be addressed; E-Mail: smhou@pku.edu.cn; Tel.: +86-10-6275-4775.

Received: 8 May 2012; in revised form: 11 June 2012 / Accepted: 12 June 2012 / Published: 25 June 2012

\begin{abstract}
We present a theoretical study of the electronic and magnetic properties of single-walled manganese phthalocyanine $(\mathrm{MnPc})$ nanotubes which can be thought of as rolled-up ribbons of the two-dimensional (2D) polymeric MnPc sheet. Our density functional theory calculations show that all of the MnPc nanotubes investigated here are half-metals with $100 \%$ spin polarization around the Fermi level. Following the increase of the tube diameter, the number of spin-down energy bands of $\mathrm{MnPc}$ nanotubes is always increased while the spin-up band gap of MnPc nanotubes approaches that of the 2D MnPc sheet in an oscillatory manner. Because the half-metallic character of MnPc nanotubes is deeply rooted in the distribution of electrons in the energy bands dominated by the Mn $3 \mathrm{~d}$ atomic orbitals, adsorption of $\mathrm{CO}$ molecules on the $\mathrm{Mn}$ ions leads to a redistribution of electrons in the Mn 3d orbitals and thus can tune precisely the spin state and electronic transport properties of $\mathrm{MnPc}$ nanotubes, demonstrating promising applications of $\mathrm{MnPc}$ nanotubes in future molecular spintronics and single-molecule sensors.
\end{abstract}

Keywords: MnPc nanotubes; half-metal; sensor; molecular spintronics; density functional theory

Classification: PACS 72.25.-b, 73.22.-f, 73.63.Fg, 85.65.+h 


\section{Introduction}

Since their discovery in 1993 [1,2], single-walled carbon nanotubes (SWCNTs) have received considerable attention due to their promising applications in nanoelectronic devices [3,4]. Depending on their chirality and diameter, metallic and semiconducting SWCNTs can be used as interconnects and conducting channels, respectively. More recently, the applications of SWCNTs have been extended to molecular spintronics [5,6]. Since infinite, pristine SWCNTs are spin degenerate, spin polarization in SWCNT-based molecular spintronic devices is always generated either by magnetic dopants decorating the tube wall [7-9] or by magnetic molecules drafted on the tube sidewall or connected to the tube end [10-14]. However, it is rather difficult to control precisely the dopant positions in the tube sidewall and the nanotube-molecule coupling. Thus, it is highly desirable to find other tubular nanostructures with intrinsic spin polarization functionalities.

Because SWCNTs can be considered as formed by rolling up the graphene sheet into a cylinder, it is a good start for the search for new tubular nanostructures from some known two-dimensional (2D) atomic sheets with novel magnetic properties. One promising candidate is the $2 \mathrm{D}$ polymeric metallophthalocyanine (MPc) sheets wherein the incorporated transition metal atoms can feature multiple functional properties, depending on their specific type and coordination environments $[15,16]$. For example, the 2D FePc polymer, which has been synthesized on both metal (Au and Ag) surfaces and a thin insulting film [15], is predicted to be an antiferromagnetic semiconductor [16]; in contrast, the ground state of the $2 \mathrm{D}$ polymeric $\mathrm{MnPc}$ sheet is determined to be half-metallic with $100 \%$ spin polarization around the Fermi level [16]. To be specific, a metallic feature appears in the spin-down channel of the 2D polymeric MnPc sheet while the spin-up channel remains semiconducting. Therefore, in this paper we investigate the electronic and magnetic properties of single-walled MnPc nanotubes which are constructed from the MnPc sheet by cutting along the (100) direction. The calculation employs spin density functional theory (DFT) within the generalized gradient approximation (GGA). Our calculations show that, different from other nanotubes such as $\mathrm{Si}, \mathrm{SiC}, \mathrm{BN}, \mathrm{AlN}, \mathrm{GaN}$ and InN which are spin degenerate [17-22], the single-walled MnPc nanotubes investigated here are all half-metals. Following the increase of the tube diameter, the number of spin-down energy bands across the Fermi level is always increased. In contrast, the size dependence of the spin-up band gap is a little complex. Although the spin-up band gap of MnPc nanotubes approaches that of the 2D MnPc sheet as the tube diameter is increased, the gap values of MnPc nanotubes with an odd number of MnPc monomers per unit cell is larger than those with an even number of MnPc monomers. Fine tuning of the electronic and magnetic properties of $\mathrm{MnPc}$ nanotubes are illustrated by adsorption of $\mathrm{CO}$ molecules on the Mn ions, which demonstrates that MnPc nanotubes have promising applications in molecular spintronic devices and single-molecule sensors.

\section{Calculation Method}

In this work we use the SIESTA code to study the atomic and electronic structures of single-walled MnPc nanotubes [23]. SIESTA is an efficient numerical implementation of DFT, in which improved Troullier-Martins pseudopotentials are used to describe the atomic cores and the GGA functional in the form proposed by Perdew, Burke and Ernzerhof (PBE) is used to account for the electron-electron 
interactions $[24,25]$. The pseudopotentials are generated using the following electronic configurations: $\mathrm{H}, 1 \mathrm{~s}^{1} ; \mathrm{C}, 2 \mathrm{~s}^{2} 2 \mathrm{p}^{2} ; \mathrm{N}, 2 \mathrm{~s}^{2} 2 \mathrm{p}^{3} ; \mathrm{O}, 2 \mathrm{~s}^{2} 2 \mathrm{p}^{4} ; \mathrm{Mn}, 4 \mathrm{~s}^{1} 3 \mathrm{~d}^{6}$. The wave functions for the valence electrons are expanded over a finite range numerical basis set and a user-defined double-zeta plus polarization (DZP) basis set is constructed for all elements including hydrogen, carbon, nitrogen, oxygen and manganese $[13,14]$. Geometry optimization is performed by conjugate gradient until the forces are smaller than $0.03 \mathrm{eV} \cdot \AA^{-1}$. The $2 \mathrm{D} \mathrm{MnPc}$ sheet is placed in the xOy plane while the MnPc nanotubes are placed along the $\mathrm{z}$ axis. In order to model isolated $\mathrm{MnPc}$ sheets and nanoutbes, the distance between neighboring MnPc sheets (nanotubes) are set to be larger than $15 \AA$.

\section{Results and Discussion}

Since single-walled MnPc nanotubes can be thought of as rolled-up ribbons of 2D MnPc sheets, we start from the atomic and band structures of the 2D polymeric MnPc sheet to understand the electronic and magnetic properties of MnPc nanotubes. As shown in Figure 1(a), the 2D MnPc polymer is a planar sheet with a square lattice, the lattice constant is calculated to be $10.71 \AA$; the Mn-N bond length is optimized to be $1.954 \AA$, very close to that of the gas-phase MnPc molecule [13]. In Figure 1(b,c) we present the spin-resolved band structure of the $2 \mathrm{D}$ polymeric MnPc sheet and the density of states (DOS) projected onto the Mn 3d atomic orbitals. As we can see, the 2D MnPc sheet has the electronic structure of a half-metal. For the spin-up electrons, the energy bands contributed by the $\pi$ and $\pi^{*}$ orbitals of the Pc ring form respectively the valence band and the conduction band around the Fermi level, giving a direct gap (at the $\mathrm{X}$ point) of $0.36 \mathrm{eV}$, while the energy bands dominated by the Mn $3 \mathrm{~d}$ orbitals are all far from the Fermi level. In contrast, for the spin-down electrons, two energy bands dominated by the $\mathrm{Mn} 3 d_{x z}$ and $3 d_{y z}$ orbitals cut across the Fermi level, resulting in a metallic character. The half-metallicity and ferromagnetism of the $2 \mathrm{D} \mathrm{MnPc}$ sheet can be explained by a charge-transfer mechanism. In brief, although an isolated $\mathrm{Mn}$ atom has an $[\mathrm{Ar}] 3 \mathrm{~d}^{5} 4 \mathrm{~s}^{2}$ electronic configuration, in the 2D MnPc sheet the Mn 4s levels are shifted above the Fermi level, and the strong crystalline field of $\mathrm{D}_{4 \mathrm{~h}}$ symmetry splits the $3 \mathrm{~d}$ shell into a doublet of $\mathrm{e}_{\mathrm{g}}\left(d_{x z}, d_{y z}\right)$ symmetry and three singlets of $\mathrm{a}_{1 \mathrm{~g}}\left(d_{\mathrm{z}^{2}}\right), \mathrm{b}_{2 \mathrm{~g}}\left(d_{x y}\right)$ and $\mathrm{b}_{1 \mathrm{~g}}\left(d_{\mathrm{x}^{2}-\mathrm{y}^{2}}\right)$ symmetry. Two of the seven valence electrons are transferred from the Mn atom to the Pc ring, and thus the central Mn ion has a $2+$ formal charge. Four spin-up electrons completely fill the energy bands dominated by the Mn $3 d_{\mathrm{xy}}, 3 d_{\mathrm{z}^{2}}, 3 d_{x z}$ and $3 d_{y z}$ orbitals, which hence are placed below the Fermi level. The remaining one valence electron only partially occupies the spin-down energy bands dominated by the $\mathrm{Mn} 3 d_{x z}$ and $3 d_{y z}$ orbitals. Consequently, the spin-down energy bands cross the Fermi level and the 2D MnPc sheet acquires a magnetic moment of $3.0 \mu_{\mathrm{B}}$ per unit cell, which is the same as that of an isolated MnPc molecule [13,14]. That is, polymerization does not change the spin state of the central Mn ion. These results obtained by using localized atomic orbitals are in good agreement with previous plane-wave DFT calculations [16]. It should be noted that the GGA + U method does not change the half-metallic characteristics of the 2D MnPc sheet though a very small in-plane shift occurs for the Mn ion [16]. 
Figure 1. The optimized geometry structure of one unit cell (a) and the spin-resolved band structure (b) of the 2D MnPc sheet; (c) DOS projected onto the Mn 3d atomic orbitals.

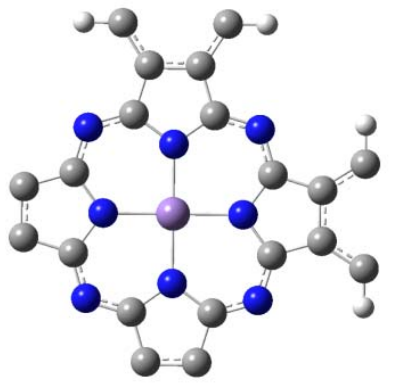

(a)

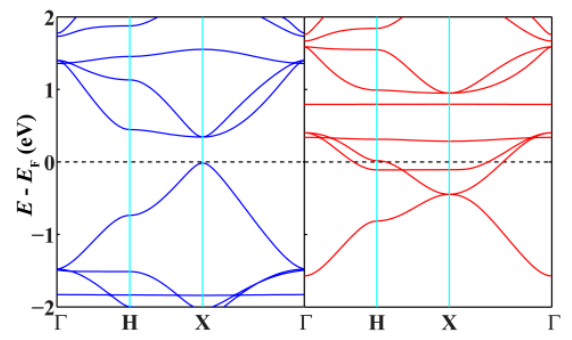

(b)

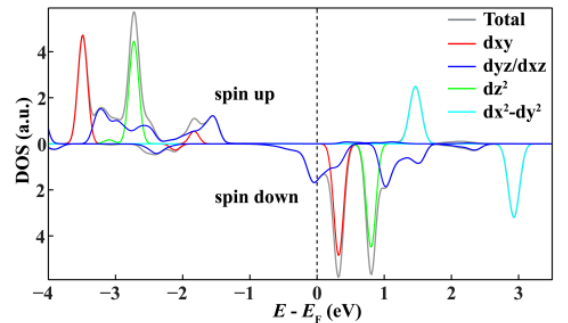

(c)

Now we move to investigate the electronic and magnetic properties of single-walled MnPc nanotubes. For the sake of brevity, these nanotubes are denoted by MnPc-n where $\mathrm{n}$ is the number of $\mathrm{MnPc}$ monomers in one unit cell. Figure 2(a) shows the optimized geometric structure of the thinnest MnPc-3 nanotube. Because the radius of the MnPc-3 nanotube is only $5.05 \AA$, the Mn-Mn distance perpendicular to the tube axis is calculated to be $8.642 \AA$ which is about $2.07 \AA$ shorter than that along the tube axis, demonstrating the large curvature of the tube wall. In order to know the effects of curvature of the tube wall, we compare the spin-resolved band structure (Figure 2(c)) of the MnPc-3 nanotube with that of the MnPc nanoribbon with the same number of MnPc monomers along its width (Figure 2(b)).

Figure 2. The optimized geometry structure of one unit cell (a) and the spin-resolved band structure (c) of the MnPc-3 nanotube, the spin-resolved band structure (b) of the $\mathrm{MnPc}$ nanoribbon with the same number of MnPc monomers along its width are given for comparison.
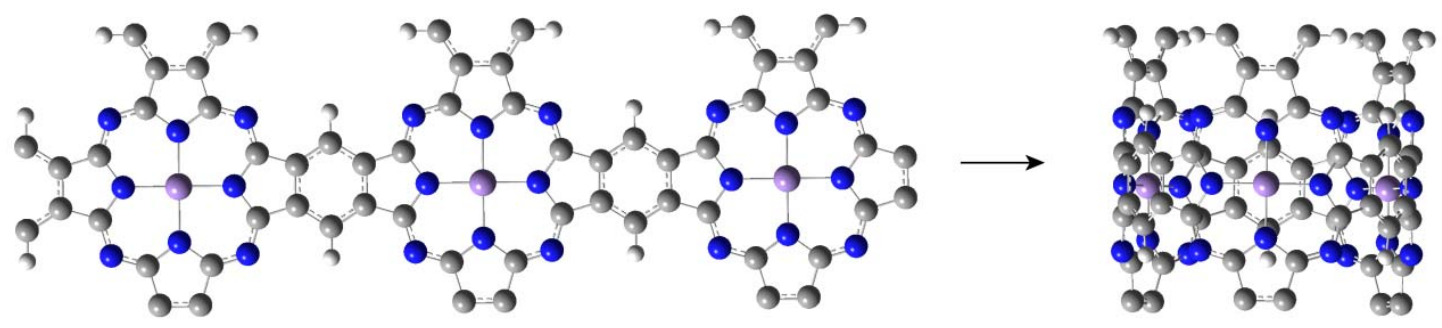

(a)

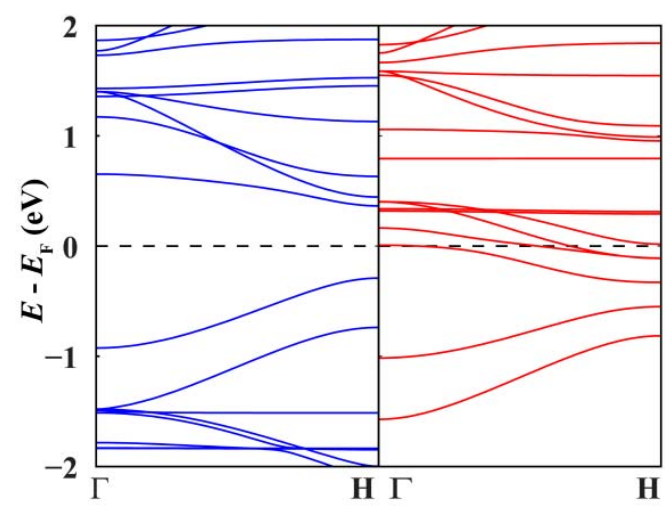

(b)

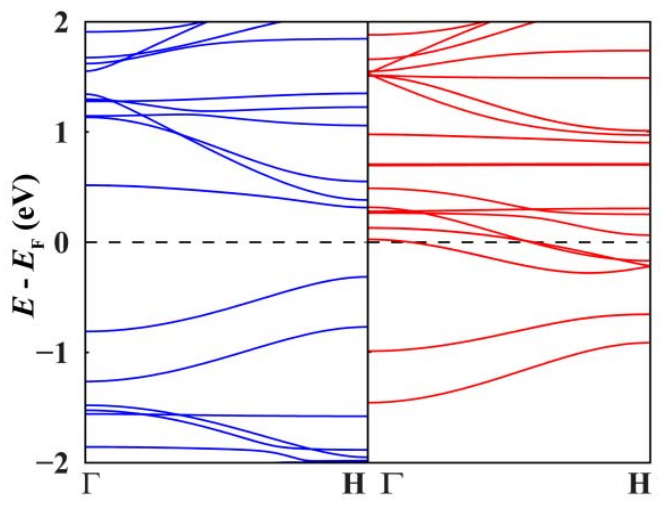

(c) 
As we can see, the differences in the overall shape of these two band structures are very small, illustrating that the large curvature of the MnPc-3 tube wall does not affect the spin state of the Mn ions significantly. This is also corroborated by the magnetic moment per unit cell of the MnPc-3 nanotube $\left(9.0 \mu_{\mathrm{B}}\right)$, which is just three times of that of the $2 \mathrm{D} \mathrm{MnPc}$ sheet. The most striking feature is that the MnPc-3 nanotube not only preserves the half-metallic characteristics but also has a larger spin-up band gap $(0.63 \mathrm{eV})$ than the $2 \mathrm{D} \mathrm{MnPc}$ sheet. For the spin-down electrons, among the six energy bands dominated by the Mn $3 d_{x z}$ and $3 d_{y z}$ orbitals five bands go across the Fermi level while another one approaches the Fermi level from higher energies at the $\mathrm{H}$ point. Thus, the ballistic transport for the infinite MnPc-3 nanotubes would be characterized by a conductance of $5 e^{2} / h$ for one of the spin channels and zero transmission for the other.

Next we investigate the size effects in MnPc nanotubes. Some important parameters characterizing the atomic and electronic structures of MnPc nanotubes are listed in Table 1, where the number of $\mathrm{MnPc}$ monomers per unit cell ranges from 3 to 6 . As we can see, all four of these MnPc nanotubes are half-metals. Unlike armchair SWCNTs which all possess two energy bands going across the Fermi level [26], the number of the spin-down energy bands of MnPc nanotubes cutting the Fermi level always increases as the tube diameter is increased. In contrast, following the increase of the tube diameter the spin-up band gap of the MnPc nanotubes does not monotonically approach that of the 2D $\mathrm{MnPc}$ sheet, because the spin-up band gaps of MnPc nanotubes with an odd number of $\mathrm{MnPc}$ monomers in one unit cell are larger than those with an even number of MnPc monomers. The stability and the synthetic possibility of the MnPc nanotubes can be evaluated by the strain energy which is determined by subtracting the total energy per atom of the 2D MnPc sheet from the corresponding values of MnPc nanotubes. As listed in Table 1, the thermodynamic stability of MnPc nanotubes increases with the increase of the tube diameter, and MnPc nanotubes with diameters larger than the MnPc-6 nanotube will have much lower strain energies $(<8 \mathrm{meV})$. That is, these MnPc nanotubes should be stable and can be rolled up easily from the 2D MnPc sheet. Although MnPc nanotubes have not been observed experimentally, our calculations indicate it is highly possible to prepare these MnPc nanotubes under appropriate experimental conditions considering that the $2 \mathrm{D}$ FePc sheet with similar structures has already been synthesized successfully [15].

Table 1. Typical parameters characterizing the atomic and electronic structures of MnPc nanotubes, where $\mathrm{D}$ is the Mn-Mn distance perpendicular to the tube axis; Es is the strain energy; Eg is the spin-up band gap; $\mathrm{N}$ is the number of spin-down energy bands across the Fermi level and $\mathrm{M}$ is the magnetic moment per unit cell.

\begin{tabular}{cccccc}
\hline & $\mathbf{D}(\AA)$ & Es $(\mathbf{m e V} / \mathbf{a t o m})$ & $\mathbf{E g}(\mathbf{e V})$ & $\mathbf{N}$ & $\mathbf{M}\left(\boldsymbol{\mu}_{\mathbf{B}}\right)$ \\
\hline $\mathrm{MnPc}-3$ & 8.642 & 35 & 0.63 & 5 & 9.0 \\
$\mathrm{MnPc}-4$ & 9.527 & 20 & 0.41 & 5 & 12.0 \\
$\mathrm{MnPc}-5$ & 9.949 & 13 & 0.48 & 7 & 15.0 \\
$\mathrm{MnPc}-6$ & 10.179 & 8 & 0.33 & 9 & 18.0 \\
\hline
\end{tabular}

Finally we investigate how to control and tune the electronic and magnetic properties of MnPc nanotubes. It is well known that the spin state of metal phthalocyanine molecules can be tuned by ligand adsorption. For example, the spin on the FePc molecule can be quenched completely or 
partially, depending on whether the ligand bonded to the iron ion is $\mathrm{CO}$ or NO [27,28]. Therefore, we choose the MnPc-3 nanotube as a representative example and study the response of MnPc nanotubes to the adsorption of $\mathrm{CO}$ on the $\mathrm{Mn}$ ions (Figure 3). The adsorption of one or two $\mathrm{CO}$ molecules does not destroy the half-metallic character of the MnPc-3 nanotube, but does decrease the number of spin-down energy bands across the Fermi level. In details, only four spin-down energy bands can go across the Fermi level when one of the three Mn ions per unit cell of the MnPc-3 nanotube is bonded to a $\mathrm{CO}$ molecule, and the adsorption of one more $\mathrm{CO}$ molecule on another $\mathrm{Mn}$ ion further decreases the number of spin-down energy bands across the Fermi level to two. Correspondingly, the magnetic moment per unit cell is also decreased from $9.0 \mu_{\mathrm{B}}$ (pristine) to $7.0 \mu_{\mathrm{B}}$ (one CO molecule) and $5.0 \mu_{\mathrm{B}}$ (two CO molecules), indicating a change of the spin state of the Mn ions. However, when the three $\mathrm{Mn}$ ions in one unit cell of the MnPc-3 nanotube are all bonded to one $\mathrm{CO}$ molecule, the spin-down conducting channels are shut down completely and the MnPc-3 nanotube is changed drastically from a half-metal to a spin-polarized semiconductor with a magnetic moment of $3.0 \mu_{\mathrm{B}}$ per unit cell. This shows an efficient way to tailor the electronic properties of $\mathrm{MnPc}$ nanoutbes: the spin state and transport properties of MnPc nanotubes can be tuned precisely by controlling the $\mathrm{CO}$ coverage.

Figure 3. (a) the optimized geometry structure of one unit cell of the MnPc-3 nanotube adsorbed with three $\mathrm{CO}$ molecules, and the spin-resolved band structures of the MnPc-3 nanotube adsorbed with one (b); two (c) and three (d) CO molecules.

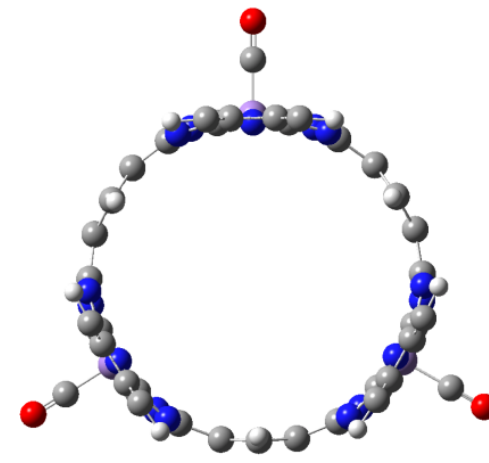

(a)

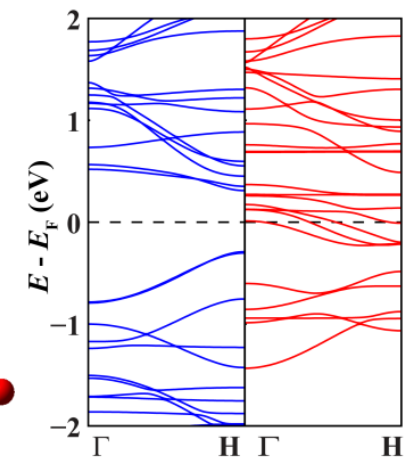

(b)

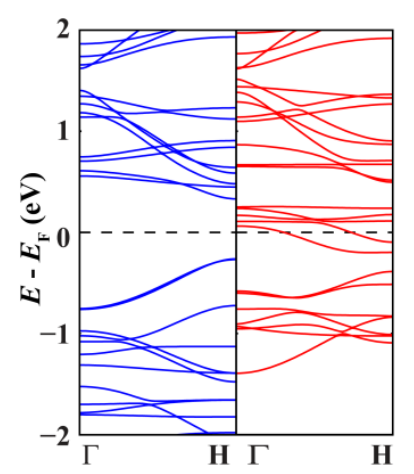

(c)

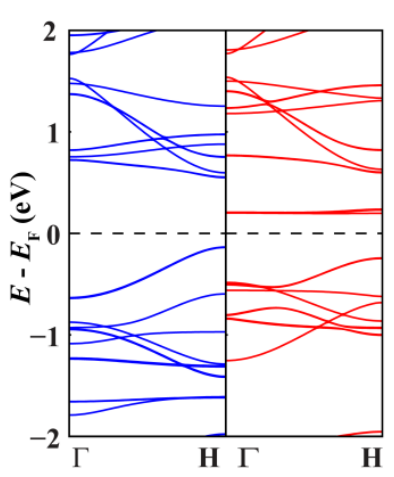

(d)

Because the curvature does not change the spin state of the Mn ions, we can analyze the mechanism of tuning the spin state of the $\mathrm{Mn}$ ions by using a simpler model, i.e., the adsorption of $\mathrm{CO}$ on the 2D MnPc sheet. As shown in Figure 4, CO binds to the Mn ion of the 2D MnPc sheet in a linear configuration, giving a binding energy of $1.08 \mathrm{eV}$. The strong interaction between the $\mathrm{Mn}$ ion and the $\mathrm{CO}$ molecule leads to a redistribution of electrons in the energy bands dominated by the $\mathrm{Mn} 3 \mathrm{~d}$ atomic orbitals resulting a change of the spin state. DOS projected onto the Mn 3d atomic orbitals shows that adsorption of $\mathrm{CO}$ empties the spin-up energy band dominated by the $\mathrm{Mn} 3 d_{\mathrm{z}^{2}}$ orbital and shifts it to $0.77 \mathrm{eV}$ above the Fermi level, leaving the spin-up energy bands dominated by the Mn $3 d_{\mathrm{xy}}, 3 d_{x z}$ and $3 d_{y z}$ orbitals still below the Fermi level. In contrast, adsorption of CO fills completely the spin-down energy bands dominated by the Mn $3 d_{x z}$ and $3 d_{y z}$ orbitals and thus moves them from around the Femi level to more than $0.62 \mathrm{eV}$ below the Fermi level. Thus, the magnetic moment per unit cell of the 2D $\mathrm{MnPc}$ sheet adsorbed with one $\mathrm{CO}$ molecule is only $1.0 \mu_{\mathrm{B}}$. Considering that the highest occupied 
molecular orbital $(3 \sigma)$ and the lowest unoccupied molecular orbital $\left(1 \pi^{*}\right)$ of CO respectively make contributions to the DOS peaks located above and below the Fermi level, we can conclude that the interaction between the CO molecule and the Mn ion in the MnPc-related structures is a typical metal-ligand bond which can be described in terms of donation of electron density from occupied molecular orbitals of $\mathrm{CO}$ and back-donation from the Mn 3d orbitals with appropriate symmetry and energy into unoccupied molecular orbitals of CO [29].

Figure 4. The optimized geometry structure of one unit cell (a) and the spin-resolved band structure (b) of the 2D MnPc sheet adsorbed with one CO molecule, and DOS projected onto the Mn 3d atomic orbitals (c) and frontier molecular orbitals of $\mathrm{CO}(\mathbf{d})$.

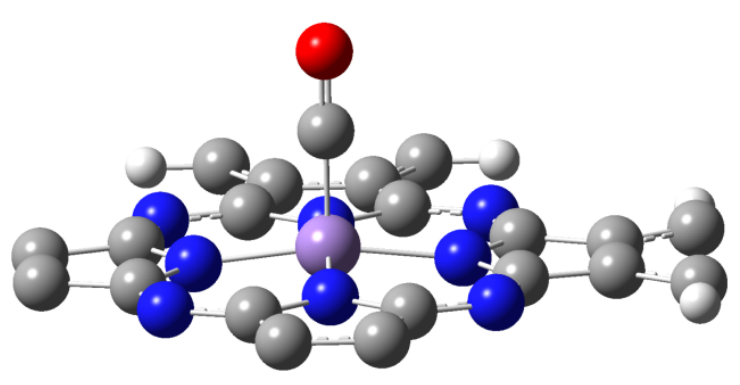

(a)

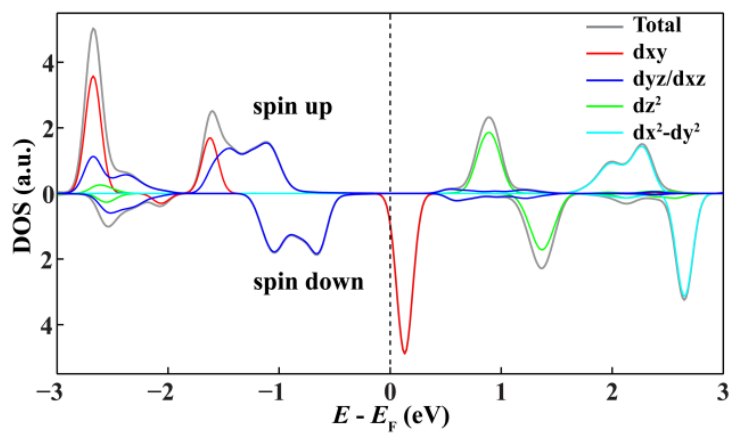

(c)

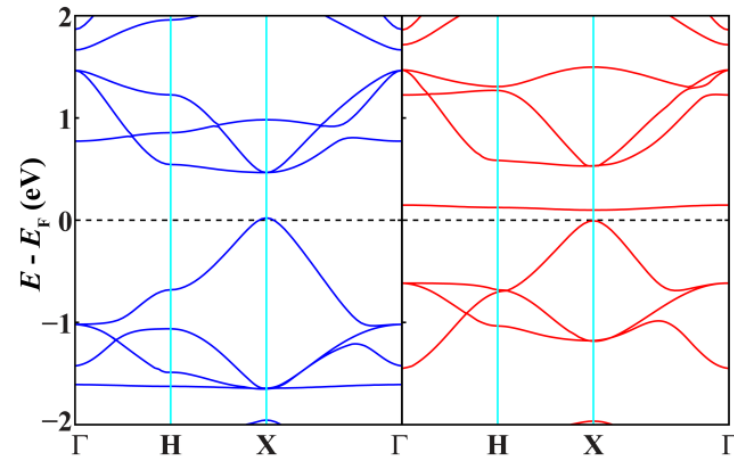

(b)

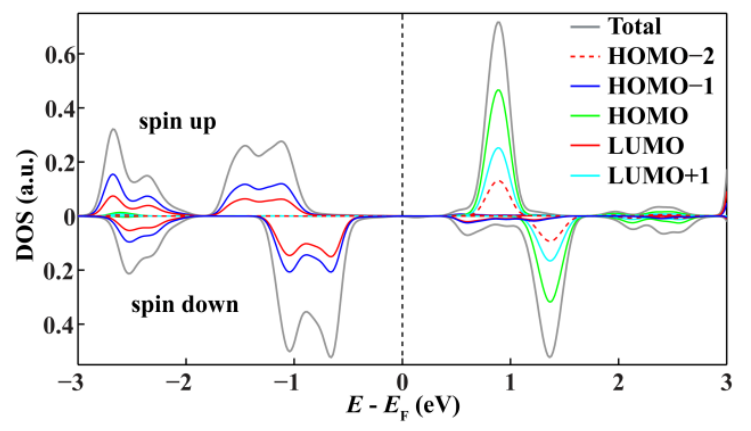

(d)

We have studied the electronic and magnetic properties of single-walled MnPc nanotubes using DFT calculations, and found that all of the MnPc nanotubes investigated here are half-metals and thus can produce $100 \%$ spin polarization around the Fermi level. As the tube diameter is increased, the number of spin-down energy bands of MnPc nanotubes is always increased while the spin-up band gap of $\mathrm{MnPc}$ nanotubes approaches that of the 2D MnPc sheet in an oscillatory manner. Because the half-metallic character of MnPc nanotubes is deeply rooted in the distribution of electrons in the energy bands dominated by the Mn 3d atomic orbitals, fine tuning of the spin state and electronic transport properties of MnPc nanotubes can be realized by adsorption of $\mathrm{CO}$ molecules on the $\mathrm{Mn}$ ions. Furthermore, other ligand molecules such as nitric oxide, pyridine and ammonia may also be able to tailor the spin state of the MnPc nanotubes, which will be pursued in future studies. Therefore, single-walled MnPc nanotubes appear as promising candidates for future molecular spintronic devices and gas sensing devices. 


\section{Acknowledgements}

This project was supported by the National Natural Science Foundation of China (No. 61071012) and the MOST of China (No. 2011CB933001).

\section{References}

1. Iijima, S.; Ichihashi, T. Single-shell carbon nanotubes of 1-nm diameter. Nature 1993, 363, 603-605.

2. Bethune, D.S.; Kiang, C.-H.; de Vries, M.S.; Gorman, G.; Savoy, R.; Vazquez, J.; Beyers, R. Cobalt-catalysed growth of carbon nanotubes with single-atomic-layer walls. Nature 1993, 363, 605-607.

3. Rutherglen, C.; Jain, D.; Burke, P. Nanotube electronics for radiofrequency applications. Nat. Nanotechnol. 2009, 4, 811-819.

4. Biswas, C.; Lee, Y.H. Graphene versus carbon nanotubes in electronic devices. Adv. Funct. Mater. 2011, 21, 3806-3826.

5. Bogani, L.; Wernsdorfer, W. Molecular spintronics using single-molecule magnets. Nat. Mater. 2008, 7, 179-186.

6. Bogani, L.; Wernsdorfer, W. A perspective on combining molecular nanomagnets and carbon nanotube electronics. Inorg. Chim. Acta 2008, 361, 3807-3819.

7. Yagi, Y.; Briere, T.M.; Sluiter, M.H.F.; Kumar, V.; Farajian, A.A.; Kawazoe, Y. Stable geometries and magnetic properties of single-walled carbon nanotubes doped with $3 d$ transition metals: A first-principles study. Phys. Rev. B 2004, 69, 075414.

8. Kirwan, D.F.; de Menezes, V.M.; Rocha, C.G.; Costa, A.T.; Muniz, R.B.; Fagan, S.B.; Ferreira, M.S. Enhanced spin-valve effect in magnetically doped carbon nanotubes. Carbon 2009, 47, 2533-2537.

9. de Almeida, J.M.; Rocha, A.R.; da Silva, A.J.R.; Fazzio, A. Spin filtering and disorder-induced magnetoresistance in carbon nanotubes: Ab initio calculations. Phys. Rev. B 2011, 84, 085412.

10. Wei, P.; Sun, L.; Benassi, E.; Shen, Z.; Sanvito, S.; Hou, S. Spin transport properties of single metallocene molecules attached to single-walled carbon nanotubes via nickel adatoms. J. Chem. Phys. 2011, 134, 244704.

11. Wei, P.; Sun, L.; Benassi, E.; Shen, Z.; Sanvito, S.; Hou, S. Effects of the covalent linker groups on the spin transport properties of single nickelocene molecules attached to single-walled carbon nanotubes. J. Chem. Phys. 2012, 136, 194704.

12. Urdampilleta, M.; Klyatskaya, S.; Cleuziou, J.-P.; Ruben, M.; Wernsdorfer, W. Supramolecular spin valves. Nat. Mater. 2011, 10, 502-506.

13. Shen, X.; Sun, L.; Benassi, E.; Shen, Z.; Zhao, X.; Sanvito, S.; Hou, S. Spin filter effect of manganese phthalocyanine contacted with single-walled carbon nanotube electrodes. J. Chem. Phys. 2010, 132, 054703.

14. Shen, X.; Sun, L.; Yi, Z.; Benassi, E.; Zhang, R.; Shen, Z.; Sanvito, S.; Hou, S. Spin transport properties of $3 \mathrm{~d}$ transition metal (II) phthalocyanines in contact with single-walled carbon nanotube electrodes. Phys. Chem. Chem. Phys. 2010, 12, 10805-10811. 
15. Abel, M.; Clair, S.; Ourdjini, O.; Mossoyan, M.; Porte, L. Single layer of polymeric Fe-phthalocyanine: An organometallic sheet on metal and thin insulating film. J. Am. Chem. Soc. 2011, 133, 1203-1205.

16. Zhou, J.; Sun, Q. Magnetism of phthalocyanine-based organometallic single porous sheet. J. Am. Chem. Soc. 2011, 133, 15113-15119.

17. Fagan, S.B.; Baierle, R.J.; Mota, R.; da Silva, A.J.R.; Fazzio, A. Ab initio calculations for a hypothetical material: Silicon nanotubes. Phys. Rev. B 2000, 61, 9994-9996.

18. Zhao, M.; Xia, Y.; Li, F.; Zhang, R.Q.; Lee, S.-T. Strain energy and electronic structures of silicon carbide nanotubes: Density functional calculations. Phys. Rev. B 2005, 71, 085312.

19. Blase, X.; Rubio, A.; Louie, S.G.; Cohen, M.K. Stability and band gap constancy of boron nitride nanotubes. Europhys. Lett. 1994, 28, 335-340.

20. Hou, S.; Zhang, J.; Shen, Z.; Zhao, X.; Xue, Z. First-principles calculations on the open end of single-walled AlN nanotubes. Physica E 2005, 27, 45-50.

21. Lee, S.M.; Lee, Y.H.; Hwang, Y.G.; Elsner, J.; Porezag, D.; Thomas, F. Stability and electronic structure of GaN nanotubes from density-functional calculations. Phys. Rev. B 1999, 60, 7788-7791.

22. Qian, Z.; Hou, S.; Zhang, J.; Li, R.; Shen, Z.; Zhao, X.; Xue, Z. Stability and electronic structure of single-walled InN nanotubes. Physica E 2005, 30, 81-85.

23. Soler, J.M.; Artacho, E.; Gale, J.D.; García, A.; Junquera, J.; Ordejón, P.; Sánchez-Portal, D. The SIESTA method for ab initio order- $N$ materials simulation. J. Phys. Condens. Matter 2002, 14, 2745-2779.

24. Troullier, N.; Martins, J. Efficient pseudopotentials for plane-wave calculations. Phys. Rev. B 1991, 43, 1993-2006.

25. Perdew, J.; Burke, K.; Ernzerhof, M. Generalized gradient approximation made simple. Phys. Rev. Lett. 1996, 77, 3865-3868.

26. Saito, R.; Dresselhaus, G.; Dresselhaus, M.S. Physical Properties of Carbon Nanotubes; Imperial College Press: London, UK, 1998.

27. Isvoranu, C.; Wang, B.; Schulte, K.; Ataman, E.; Knudsen, J.; Andersen, J.N.; Bocquet, M.L.; Schnadt, J. Tuning the spin state of iron phthalocyanine by ligand adsorption. J. Phys. Condens. Matter 2010, 22, 472002.

28. Isvoranu, C.; Wang, B.; Ataman, E.; Knudsen, J.; Schulte, K.; Andersen, J.N.; Bocquet, M.L.; Schnadt, J. Comparison of the carbonyl and nitrosyl complexes formed by adsorption of $\mathrm{CO}$ and NO on monolayers of iron phthalocyanine on Au(111). J. Phys. Chem. C 2011, 115, 24718-24727.

29. Miessler, G.L.; Tarr, D.A. Inorganic Chemistry, 4th ed.; Prentice Hall: Upper Saddle River, NJ, USA, 2010.

(C) 2012 by the authors; licensee MDPI, Basel, Switzerland. This article is an open access article distributed under the terms and conditions of the Creative Commons Attribution license (http://creativecommons.org/licenses/by/3.0/). 